can and should be seen by appointment. The medical officer should be assisted by clinical assistants and a system of promotion should be introduced.

(8) Laboratories run for this service should be staffed by properly trained persons who have had special experience in the practice of venereal diseases pathology.

These, then, are the main objects which I think we all should consider seriously. Dr. Burgess will give us the point of view of a medical officer engaged exclusively in public health work and I shall be extremely interested to hear what proposals he has to make.

\title{
Now is the time for action
}

Before I conclude, I should like to lay stress on the fact that if we wish to raise the standard of work in our speciality and the status of those engaged in it, now is the time to do so. I feel certain that if as a society we take no action now, we shall lose ground after the war and be regarded as of little or of no account. After the discussion therefore I shall propose the formation of a subcommittee for the purpose of formulating a policy for the conduct of the venereal diseases scheme.

\section{IS A NEW DEAL IN THE CONTROL OF VENEREAL DISEASE NECESSARY ?-2*}

\section{By J. A. BURGESS, M.D., D.P.H.}

Chief Venereal Diseases Officer and Assistant County Medical Officer of Health, West Riding, Yorkshire

In the introduction to the first number of The British Journal of Venereal Diseases it was stated that this Society confines its activities to the medical study of venereal diseases. Today's subject for discussion is probably one of the few exceptions to this rule. In advocating a new deal in venereal disease control, one must take into consideration also the administrative, social and moral aspects. It is right that we should break away from our study of the clinical effects of venereal diseases from time to time and ask ourselves whether we are in fact doing everything possible to deal with their menace.

In the first place, I do not wish to give the impression that I think our V.D. service is not a good one-far from it. Splendid work has been and is being accomplished. Some of us take the view however that improvements are necessary if we are to have a V.D. scheme which is second to none. In addition, more attention must be paid in the future to the prevention of these diseases.

Although a medical officer of health may be keenly desirous of providing his district with the best possible service, his activities are often limited by extraneous circumstances and the final decision on any matter rests with his committee. The individual members of the committee, having no practical experience of the work done at V.D. clinics and little more knowledge of venereal disease than the average layman, cannot be expected to assess the position to the best advantage. The result is that undue weight is often attached to the financial implications. Unfortunately, venereal disease has been a comparatively neglected branch of the public health services. For example, the Society of Medical Officers of Health has a dental officers' group, a fever hospital group, a maternity and child welfare group, a school medical service group and a tuberculosis group, but it has no venereal diseases group !

I understand that, under the block grant system, authorities are provided annually with a sum of money the amount of which is reviewed at the end of each five-year period. This sum goes toward the combined costs of the various public health services. Is it not possible then that one branch which is unpopular or to which some stigma is attached may receive less consideration than the others?

\footnotetext{
* An address to the Medical Society for the Study of Venereal Diseases, 27th November, 1943.
} 
In addition, each clinic operates as an individual unit and apart from the itinerant patient's treatment booklet (V.15) there is no connecting link between one V.D. clinic and another. Incidentally, it must be disconcerting to travelling patients to find that methods of treatment vary considerably from clinic to clinic.

To overcome these drawbacks, I support Dr. Price's suggestion that there should be a National Venereal Diseases Service controlled from one point, with subdivisions all over the country. Each scheme-making authority would require to have some control in the administration of the service in its own area and the medical officer of health, with his knowledge of local conditions, would act in an advisory capacity. The service as a whole however would be under the direction of V.D. experts with extensive clinical and administrative experience. Other benefits of such a scheme would include the provision from a central pool of trained venereologists who temporarily could take over clinics during the absence of the medical officer on account of sickness, of holidays or of refresher training.

\section{Venereal disease education}

One of the first steps we must take toward the control of venereal disease is to eliminate completely the Victorian priggishness in dealing with sex with which we as a nation have so long been afflicted. We have gone some way toward this goal during the past year and as recently as three weeks ago the Board of Education published a pamphlet advocating instruction to school children on sex and moral behaviour. This is certainly an advance but the full advantage of the work will not be evident for at least a decade.

Most of us will agree that the venereal diseases propaganda carried out by the Central Council for Health Education and the Ministry of Health during this year has done an immense amount of good. The truth has been told but not, I regret to say, the whole truth. The newspaper V.D. advertisements, quite rightly, state in effect, " no promiscuity, no venereal disease." But what of the thousands who have been foolish enough to disregard this warning? Are they to await the development of some symptom or sign before seeking advice ? The public are aware that there are preventive measures which can be taken, and for us entirely to ignore this important fact is to make us akin to the ostrich who buries its head in the sand. It would have to be pointed out that for various reasons including the influence of alcohol, the time factor and the site of inoculation, self-applied prophylaxis is often unavailing and that in any event it is not 100 per cent efficient.

Furthermore, all persons who have exposed themselves to the risk of infection should be advised to go to a special treatment centre at the very earliest opportunity. In the case of some patients the medical officer could apply effective preventive treatment and in the case of the remainder could keep them under observation during the incubation period and until satisfactory tests had been performed.

I cannot believe that if the general public were told the truth about V.D. prophylaxis there would be any increase in immorality. My experience with V.D. patients leads me to the conclusion that few of them fear venereal disease when a risk of infection is taken. This contention is supported by the fact that the number of persons who attend clinics for preventive treatment within a few hours of exposure to infection is an infinitesimal fraction of the total number of new cases.

\section{Medical Profession}

The busy general practitioner is not entirely to blame for the annoying errors in diagnosis and treatment which sometimes come to light in patients referred to us. As a student his training in venereal diseases. is the barest minimum and because many patients go direct to a clinic he sees comparatively few V.D. cases in his work. Refresher courses for practitioners are probably out of the question at the present time, but some form of postgraduate instruction in venereology is necessary.

Many of you will have seen the excellent talking film produced by the Ministry of Information under the direction of the Ministry of Health on the diagnosis and 
treatment of scabies. A similar type of film dealing with early syphilis and gonorrhoea is urgently required and would be appreciated by most medical men.

Even amongst V.D. clinio medical officers there are to be found those whose interest in this subject is entirely superficial and sometimes only financial. I suppose that a few men of this type are to be found in all branches of Medicine but they seem to be unduly prevalent in V.D. work. This may be the result of the ease with which a V.D. Certificate can be obtained-merely by 130 hours of training at a large V.D. clinic. Any doctor with three or four weeks to spare can acquire the qualifications required for the position of venereal diseases officer. In my view a much longer period of training is essential and this should include several months in an approved laboratory.

To raise the general standard of V.D. work I suggest that there should be a Diploma of Venereology, preferably promoted by this Society. Ultimately, the possession of such a diploma might become a sine qua non for the position of medical officer in charge of a special treatment centre.

A factor which mitigates against providing the best possible venereal diseases service is the low scale of remuneration offered for whole-time appointments. To the keen and ambitious young doctor venereology is a blind alley with few chances of promotion to a four-figure salary even after fifteen or twenty years' whole-time experience and the possession of a string of additional medical qualifications.

We have still a great deal to learn about this subject and grants of money are required for research. A Chair of Venereology might well be endowed at one of our universities. I should be interested to learn from our American colleagues what the position in the United States is in these respects.

\section{Compulsory methods}

We have had experience of Regulation 33B for less than one year, hence it is with some hesitation that I comment on its value. Briefly I consider it to have been a step in the right direction but it does not go far enough. In my area the compulsory powers under 33B have been applicable to less than one per cent of the total number of new cases of early syphilis and gonorrhoea. The Regulation has resulted however in seventy-two persons suspected to be suffering from venereal disease attending voluntarily at a special clinic for examination. This number represents approximately five per cent of the total number of new cases seen at the various clinics.

I appreciate the point of view of those who maintain that further compulsory powers might have the effect of driving venereal diseases underground. It is extremely difficult to refute this argument. One might point out that much V.D. is already underground and that the spadework performed by voluntary methods has not dug deeply enough to reveal it. Even before the war there were many thousands of new infections discovered at our clinics each year coupled with a high defaulter rate. For example, in the West Riding in 1938 the ratio of early syphilis defaulters to new cases of early syphilis was $58: 100$.

The danger to the community of defaulting early syphilitics must not be minimized. These patients belong to the group who in another ten or fifteen years may be occupying valuable bed space in our hospitals, quite apart from the danger of infecting others in the earlier years and of procreating congenital syphilitics.

Compulsory schemes function satisfactorily in various other countries but this fact is, by itself, a relatively weak argument for their introduction here. On the other hand I understand that members of the Forces suffering from venereal disease are required to undergo treatment and that the concealment of V.D. is a punishable offence. We are not all in the Forces but most of us have accepted some form of conscription without demur and fundamentally in war-time the viewpoint of the soldier and of the civilian cannot be poles apart. In at least one respect V.D. patients in the Forces would appear to have even more reason to conceal their infection than would civilians. I am referring to the fear that leave, promotion or pay, might be adversely affected.

While the present state of public enlightenment on venereal disease in this country is probably such as to make the introduction of a fully compulsory 
scheme impossible, nevertheless measures might be taken to deal with the most serious menace, namely early syphilis. This could be done by an Order under the Defence Regulations giving medical officers of health of scheme-making authorities power to require persons, whom they had reason to believe might be infected with syphilis in a communicable form, to present themselves for examination by a special practitioner within a specified number of days. In addition, all persons found to be suffering from primary or secondary syphilis could be obliged to undergo treatment by a special practitioner until he was satisfied that the patient was free from the disease.

\section{Venereal disease clinics}

A factor which contributes towards the difficulties of bringing the maximum number of infected persons under treatment is the unsuitable situation and poor design of the clinics. If we are to convince the public that "V.D. is a disease and not a disgrace" we must provide treatment centres which are brighter, cleaner and better equipped than those of out-patient departments generally. V.D. clinics should always be held at hospitals or at health centres and should be so arranged that patients could enter the clinic waiting room without other persons knowing which department they were attending. We tell patients that the strictest secrecy will be observed yet there are clinics where the consulting room is far from sound-proof and conversations between doctor and patient can be overheard by those in the-waiting room. Instead of a single large waiting room, it would be better to have a number of small private waiting cubicles. This would reduce the number of chances of patients seeing acquaintances while waiting.

It might be wise if the term V.D. clinic were abandoned altogether and venereal diseases treated under the cloak of the genito-urinary or gynaecological clinic. I am not suggesting that the treatment of venereal diseases should be taken over by genito-urinary surgeons and gynaecologists but when new out-patient departments are being considered, the female V.D. and gynaecological clinic premises might be combined, and similarly the male V.D. and genito-urinary clinic.

Representations have been made in some quarters that more V.D. clinics are required. This may be the case but the establishment of more clinics is certainly not the whole solution to the problem of the control of venereal disease. I do know that in the smaller townships with a population of 20,000 or so a V.D. clinic is not always a success ; patients prefer to go to another town where there is less chance of being $r \in \operatorname{cognized}$. Probably every town of a population of 40,000 and over should have V.D. clinic facilities.

Not only must the clinic hours be arranged so as to suit the convenience of patients but there also must be a sufficient number of sessions so that the work can be carried out thoroughly and not under conditions in which speed is allimportant. A medical officer should not be expected to examine and treat more than from 12 to 15 patients per hour.

\section{Venereal disease tests in relation to maternity}

Many maternity and child welfare authorities do not appreciate sufficiently the value of blood tests during pregnancy. In those districts where antenatal blood testing is a routine procedure the number of cases of syphilis discovered varies between less than 1 and 7 per cent.

Berger (1943) has described an agglutination test for the serological diagnosis of syphilis which he claims has a 100 per cent sensitivity compared with 66 and 67 per cent for the Kahn and Wassermann tests respectively. My clinical experience with this test indicates that although a few false positive reactions occur there are no false negatives. For this reason it would be a valuable blood serum test to do on specimens from antenatal patients. A diagnosis of syphilis could not be made on a positive agglutination test alone ; but it would focus attention on those cases requiring further investigation and no prospective mother with syphilis would have her infection undiscovered. A gonococcal complement fixation test should also be done on each routine sample of blood serum ; in the same way 
a positive result would be an indication for a thorough examination to exclude gonorrhoea.

It has always been a matter of surprise to me that medical officers of health whose chief concern is with the preventive aspect of medicine have not taken more advantage of the ophthalmia neonatorum notifications to trace untreated cases of gonorrhoea. About 50 per cent of cases are said to be due to the gonococcus but no financial or other stimulus is given to general practitioners to take smears or cultures (or smears and cultures) of the eye discharge in order that those due to the gonococcus can be identified. If a small fee were payable to doctors for doing this work the mothers of the gonococcal cases could be visited and be persuaded to attend a special clinic for examination.

Finally, I realize that there are a number of V.D. control measures which have been advocated from time to time and which I have not mentioned. I have done this not because these methods are of little importance but because I have had insufficient experience of their mode of operation to come to any decision on their value. I should like to add this however, that if we are ever to achieve adequate control of venereal disease now is the time for action when public interest in its prevalence has been awakened out of the lethargic state in which it has lain for the past twenty years.

\section{REFERENCES}

Berger, F. M. (1943) J. Path. Bact., 55, 363.

\section{DISCUSSION ON THE PRECEDING PAPERS}

Col. L. W. Harrison, who opened the discussion, said :

"I have asked to be allowed to speak early in this discussion because I believe that many people are not at all clear about the powers of the Ministry of Health in regard to appointments and arrangements generally under the V.D. scheme. At least I judge this from the numbers of people who come to me asking me to give them appointments as V.D. officers. First of all, to dispel any impression which might be created by Dr. Price's remarks that our V.D. arrangements were the world's worst, I should like to say that I have visited clinics in a number of countries both on this side of the Atlantic and on the other and, by and large, I think that our clinics are as good as the others. I do not say that our best is the best in the world, or any nonsense of that kind, but the average of ours, with all their imperfections-and nobody is more conscious of the imperfections of our clinics than I am-is as good as the average elsewhere.

"Except in regard to Dr. Price's remarks about almoners-and I will leave that subject to others-I agree with much that has been said by the openers of this discussion but $I$ propose to show that we could not approach the ideal we want unless either: (a) hospital staffs, local authorities and all concerned with appointment of V.D.O.'s and with all other arrangements which go to the making of successful clinics, design of premises and so forth, are educated to the same pitch of keenness as ourselves; or $(b)$ the whole organization and administration of the V.D. scheme is centralized in the Ministry of Health.

"I will explain by examples. Prior to the coming into operation of the Local Government Act of 1929 (in April, 1930) the Ministry of Health exercised a fairly close supervision and medical officers of the Ministry made routine inspections of clinics. The inspections were necessarily infrequent because there were only three or four medical officers available for the work and I calculate that it would have taken one M.O. from two to three years to inspect all the clinics in this country if the visits were timed to coincide with sessions ; but they did keep us much more closely in touch with the work than after 1929. Appointments of medical officers were mostly in the hands of the staffs of the hospitals at which the clinics were situated and very often the medical officer appointed was a member of the staff, or someone who was persona grata with the staff, rather than the best person available in the area. The approval of the Ministry of Health was necessary but it was often difficult to find specific objections to a choice although local information might suggest that it was not the best that could have been made. Frequently hospitals refused to appoint any nominee of the local authority, although he might have been obviously the best man for the work and frequently when cogent criticisms were made of the medical officer's work, his colleagues supported him through thick and thin. Very loyal and all that but not making for efficiency. The fact is that very often hospital staffs do not regard V.D. work as requiring any special skill and experience. When I have said emphatically that for a given appointment the medical officer should be someone whose prime interest is venereal diseases, members of the hospital staff attending the conference for discussion of the arrangements of a clinic have exclaimed that surely I would not expect a medical officer to confine his interest to V.D. ; how narrowing ! And so forth. They had not studied Osler on the subject of syphilis. The main point is that people who are not aware of the mental equipment necessary in a good V.D.O. are not the people to be entrusted with the choice of one. 